\title{
Aberration-Corrected STEM Imaging and EELS Analysis of Extraterrestrial Nanodiamond-Glassy Carbon Residues
}

\author{
R.M. Stroud* and M. F. Chisholm** \\ * Materials Science and Technology Division, Naval Research Laboratory, Washington, DC 20375 \\ ** Materials Science and Technology Division, Oak Ridge National Laboratory, Oak Ridge, TN \\ 37831-6069
}

Nanodiamond residues extracted from meteorites carry noble gas impurities with isotopic compositions indicative of an extrasolar origin [1]. However, the 2-nm average size of the nanodiamonds precludes isotopic analysis of individual particles, and thus the origin remains controversial. Multiple spectroscopic studies [1-3] of meteoritic nanodiamond residues have indicated that an additional, non-diamond component comprises up to $50 \%$ of the residues. Previously, this was attributed to surface reconstruction of the nanodiamonds [4] or contamination. However those measurements were limited to probe sizes $(>1-\mathrm{nm})$ at which the surfaces were not well resolved from the particle cores. By application of aberration-corrected scanning transmission electron microscopy (STEM) with an $\sim 0.15 \mathrm{~nm}$ probe, we demonstrate that nanodiamond residues from two meteorites in fact contain a second phase of carbon. On the basis of the local atomic structure, C K edge and low-loss electron energy-loss spectroscopy (EELS) data and acid-resistance, we identify this phase as glassy carbon. Our analyses also reveal numerous impurities, and we plan to refine our methods in order to identify the host phase of the noble gas atoms.

Two nanodiamond isolates were examined: the Allende DM separate first analyzed by Lewis et al. [1], and a new residue of the Murchison meteorite [5]. The samples were drop cast onto lacey carbon, ultrathin-carbon-backed lacey carbon, and 5-nm thick amorphous Si membranes that were first cleaned with argon plasma. The use of multiple types of support film allowed us to directly compare our studies with prior results, to optimize the imaging contrast for single-atomic-plane variations in sample thickness and to minimize the chance of contamination of the samples with terrestrial carbon. The samples were first characterized with a JEOL 2200FS field emission TEM at the Naval Research Laboratory, and subsequently analyzed with the Nion UltraSTEM at Oak Ridge National Laboratory. Medium angle dark field (MADF) images and EELS spectra were obtained with the UltraSTEM operating at $60 \mathrm{kV}$.

The presence of a second phase of carbon is readily apparent in the conventional bright field TEM images of the samples supported on 5-nm Si membranes (Fig. 1). It can also be seen on the ultrathin carbon supports, but is not readily observed over the large holes in lacey carbon due to particle aggregation.

MADF images reveal the structure of the nanodiamond and glassy carbon, and also abundant impurity atoms (Fig. 2). The glassy carbon consists of disordered, wrinkled sheets of 5, 6 and 7 member carbon rings. The identity of the impurity atoms can be determined from the image intensity by use of the relationship $\mathrm{I} \propto \mathrm{Z}^{1.64}$, and calibration of the proportionality factor for the specific imaging conditions using the quantized thickness variations of the sample. The observed impurities range from $Z=9(\mathrm{~F})$ to $Z=26(\mathrm{Fe})$ with an uncertainty of $\sim \pm 1$. The majority of these impurities are likely products of the acid dissolution of the meteorites used to produce the residues. The motion of 
the impurities, visible during scanning and captured as a movie, demonstrates that most are surface adsorbed, rather than structurally bound to either the diamond or glassy carbon. Due to the low overall abundance, no impurities with an image intensity corresponding to Xe or other noble gasses related to a supernova origin have yet been detected.

Carbon K edge EELS spectra were obtained with a $\sim 0.15 \mathrm{~nm}$ probe size as 100 -pt line scans spanning the glassy carbon and nanodiamond (Fig. 3). The spectra from the nanodiamond reveal $\mathrm{sp}^{3}$ bonding consistent with that of bulk diamond, with the exception of a small $\pi^{*}$ peak at $284.3 \mathrm{eV}$. This peak has previously been interpreted for nanodiamonds as a surface reconstruction feature, however in this case it may simply be due to wetting of the top and bottom nanodiamond surfaces with glassy carbon. The spectra from the glassy carbon are consistent with disordered $\mathrm{sp}^{2}$ bonded carbon. Low loss EELS spectra of the nanodiamond and glassy carbon regions show a shift in the plasmon peak position indicative of varying contributions of these two phases.

References

[1] R. S. Lewis et al., Nature 326 (6109), 160-162 (1987).

[2] D. F. Blake et al., Nature 332 (6165), 611-613 (1988).

[3] T. J. Bernatowicz, P. C. Gibbons, and R.S. Lewis, Astrophys. Jour, 359 (1), 246-255 (1990).

[4] L. A. J. Garvie, Meteor. \& Planet. Sci. 41 (5), 667-672 (2006).

[5] R. M. Stroud et al., Proceed. of the Lunar and Planet. Sci. Con., Abstract \#1940 (2011).

[6] RMS thanks C.M. O'D. Alexander and P. Heck for providing samples. This work was supported by the Office of Naval Research, NASA and the Materials Science and Engineering Division of the Department of Energy.
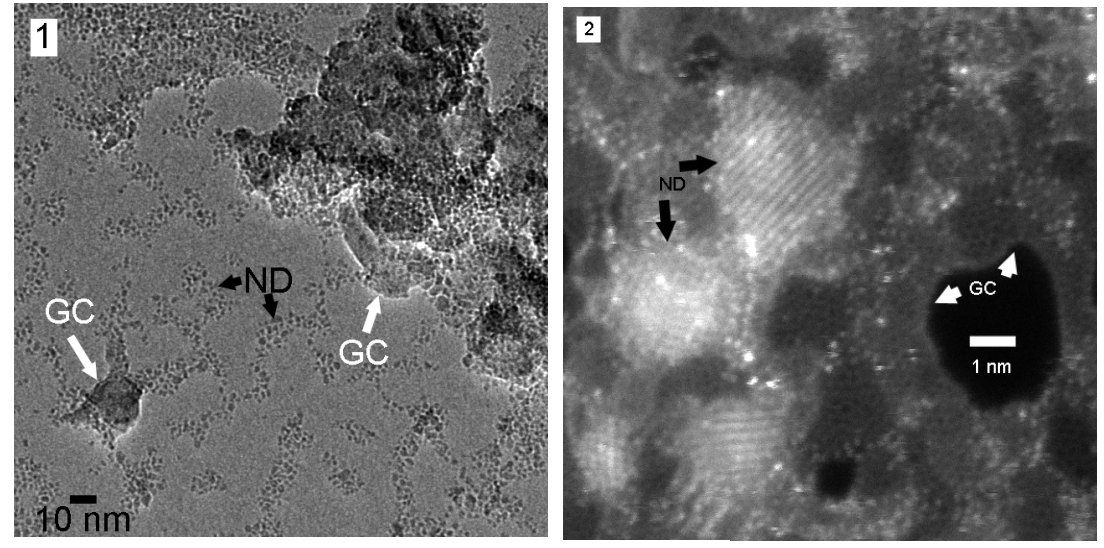

FIG. 1. Bright field TEM image of nanodiamond (ND) and glassy carbon (GC) on a 5-nm thick amorphous silicon membrane.

FIG. 2. MADF STEM image of nanodiamonds and glassy carbon, revealing abundant impurity atoms.

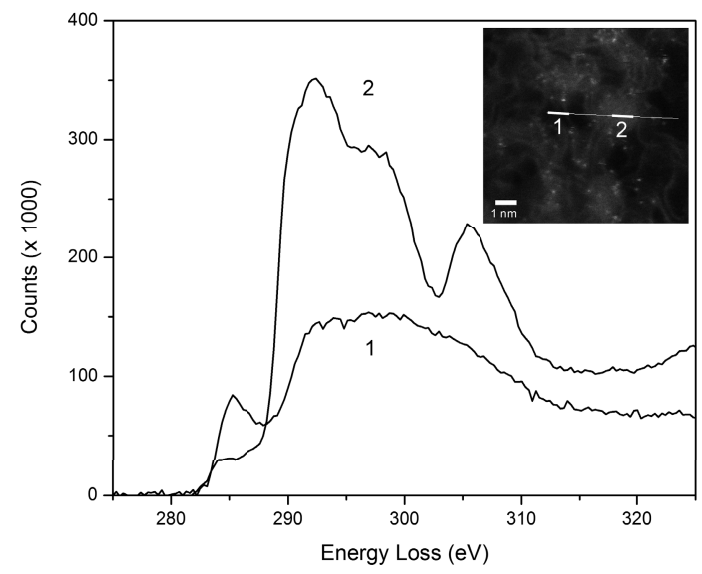

FIG. 3. C K edge EELS spectra from glassy carbon (1) and nanodiamond (2). The spectra were obtained as a linescan spectrum image. The extracted spectra are summed over 6 points each, as indicated on the inset image. 